\title{
Medical Image Segmentation using Penalized FCM and Pollination based Optimization Approach
}

\author{
Husanjot Kaur \\ Dept Of Computer Science \\ Purcitm, Mohali.
}

\author{
Rekha Bhatia \\ Dept Of Computer Science \\ Purcitm, Mohali
}

\begin{abstract}
Image segmentation is the process of partitioning a digital image into multiple segments (sets of pixels, also known as super pixels). The goal of segmentation is to simplify or change the representation of an image into something that is more meaningful and easier to analyze. Image segmentation is typically used to locate objects and boundaries (lines, curves, etc.) in images. In this paper we are going to discuss various approaches in image segmentation and various segmentation algorithms in image processing and analysis.
\end{abstract}

\section{General Terms}

Image segmentation, Pollination based Optimization, Penalized FCM.

\section{Keywords}

Image Segmentation, Medical Images, Pollination, Fuzzy CMean, Optimization.

\section{INTRODUCTION}

The division of a picture into significant structures, picture division, is frequently a crucial venture in picture examination, object representation, visualization, and numerous other picture handling undertakings. We have concentrated on the best way to examine and speak to an article, yet we accepted the gathering of pixels that recognized that protest was known in advance. In this section, we will concentrate on techniques that find the specific pixels that make up an article. An awesome assortment of division systems has been proposed in the previous decades, and some order is important to present the techniques appropriately here. A disjunction order does not appear to be conceivable however, on the grounds that even two altogether different division methodologies may impart properties that oppose particular categorization1. The classification exhibited in this part is subsequently rather an order with respect to the accentuation of a methodology than a strict division.

The following categories are used:

\subsection{Threshold based segmentation:-}

Histogram thresholding and cutting methods are utilized to section the picture. They may be connected specifically to a picture, yet can likewise be consolidated with pre and postpreparing systems. In thresholding pixels are dispensed to classes as indicated by the scope of qualities in which first shows limits which were gotten by thresholding the muscle picture. Pixels with values fewer than 128 have been put in one classification, and the rest have been put in the other class. The limits between contiguous pixels in diverse classifications have been superimposed in white on the first picture. It can be seen that the edge has effectively fragmented the picture into the two dominating. Thresholding is most likely the most often utilized procedure to portion a picture. The thresholding operation is a dark quality remapping operation g characterized by:
$\mathrm{G}(\mathrm{v})=0$ if $\mathrm{v}<\mathrm{t}, 1$ if $\mathrm{v}>\mathrm{t}$,

Where $\mathrm{v}$ speaks to a dark quality and $\mathrm{t}$ is the limit esteem. Thresholding maps concur esteemed picture to a double picture. After the thresholding operation, the picture has been fragmented into two portions, recognized by the pixel values 0 and 1 separately. In the event that we have a picture which contains splendid questions on a dim foundation, thresholding can be utilized to section the picture. Since in numerous sorts of pictures the dim estimations of articles are altogether different from the foundation worth, thresholding is regularly an appropriate strategy to fragment a picture into items and foundation. In the event that the items are not covering, then we can make a different fragment from every item by running a naming calculation on the limit parallel picture, in this way appointing a remarkable pixel worth to every article. Numerous techniques exist to choose suitable edge esteem for a division errand. Maybe the most widely recognized strategy is to situate the limit esteem intelligently; the client controlling the quality and assessing the thresholding result until a fulfilling division has been acquired. The histogram is regularly an important device in making suitable edge esteem.

\subsection{Edge based segmentation:-}

Since a (paired) item is completely spoken to by its edges, the division of a picture into divided articles can be accomplished by discovering the edges of those items. A run of the mill way to division utilizing edges is (1) figure an edge picture, containing all(plausible) edges of an unique picture, (2) process the edge picture so just shut item limits remain, and (3) change the outcome to a standard sectioned picture by filling in the article limits. The edge location step (1) has been examined. The third step, the filling of limits, is not a troublesome step, and a case of how this can be attained to is given for the system underneath. The trouble regularly lies in the center step: changing an edge (or edge-ness) picture to shut limits frequently requires the evacuation of edges that are brought about by commotion or different antiquities, the connecting of holes at areas where no edge was recognized (yet there ought to coherently be one) and insightful choices to associate those edge parts that make up a solitary article. The segment nervous connects beneath locations some of these difficulties. The next segments on watershed division and dynamic shapes manage systems that abstain from needing to connection edge parts by controlling -like an elastic band- forms that are constantly shut until they best fit an edge picture. In those uncommon situations where an edge picture as of now shows immaculate shut item limit.

\subsection{Region based segmentation:-}

Articles were found by placing their limits. In this segment, author talk about the double approach of discovering the item area rather than its edges. In principle, discovering an item by finding its limit and discovering it by making the area it covers will provide for you precisely the same article; the limit and the district are simply distinctive representations of the same article. On the other hand, taking an edge based way 
to division may give drastically diverse results than taking a locale based methodology. The explanation behind this is that we are certain to utilizing blemished pictures and defective strategies, subsequently the pragmatic consequence of spotting an item limit may be not the same as finding its district. Locale based division systems have just two fundamental operations: part and combining, and numerous routines even peculiarity one and only of these. The essential way to picture division utilizing fusing is.

1. Obtain an initial (over)segmentation of the image,

2. Merge those adjacent segments that are similar -in some respect- to form single segments,

3. Go to step 2 until no segments that should be merged remain.

The initial segmentation may simply be all pixels, i.e., each pixel is a segment by itself. The heart of the merging approach is the similarity criterion used to decide whether or not two segments should be merged. This criterion may be based on grey value similarity (such as the difference in average grey value, or the maximum or minimum grey value difference between segments), the edge strength of the boundary between the segments, the texture of the segments, or one of many other possibilities. The basic form of image segmentation using splitting is:

1. Obtain an initial (under)segmentation of the image,

2. Split each segment that is inhomogeneous in some respect (i.e., each segment that is unlikely to really be a single segment).

\section{Go to step 2 until all segments are homogeneous}

The introductory division may be no division whatsoever, i.e., there is just a solitary fragment, which is the whole picture. The rule for in homogeneity of a section may be the fluctuation of its dark values, the difference of its surface, the event of solid inside edges, or different other criteria. The fundamental consolidating and part systems see to be the topdown and base up way to the same system for division, yet there is an inborn contrast: the blending of two sections is direct, yet the part of a section obliges we build suitable subportions the fragments can be part into. Fundamentally, regardless we have the division issue we began with, aside from it is currently characterized on a more nearby level. To stay away from this issue, the fundamental part approach is regularly upgraded to a joined part and union methodology, where inhomogeneous sections are part into basic geometric structures (as a rule into four squares) recursively. This obviously makes discretionary fragment limits (that may not be associated to sensible limits), and union steps are incorporated into the procedure to uproot inaccurate limits.

\section{RELATED WORK}

The authors of [1] introduced an effective CNN based segmentation method with lung and brain MRI images. Authors take two steps: 1)Pre-processing of the brain and lung images, 2) Segmentation using cellular neural network in the preprocessing step, picture de-noising is carried out utilizing the direct smoothing channels, for example, Gaussian Filter. At that point, the preprocessed picture is sectioned by based picture division. At last, the distinctive MRI pictures (mind and lung) are given to the proposed way to assess the execution of the proposed approach in division process. The Comparative examination is done Fuzzy C-implies (FCM) and K-implies order. The proficiency is attained to with mind and lung MRI pictures. The Comparative examination is done with Fuzzy C-implies (FCM) and K-implies grouping. From the near examination, the precision of proposed division methodology creates preferred results over that of existing Fuzzy C means (FCM) and K-implies characterization.

The authors in [2] proposed fuzzy connectedness picture division for restorative picture recovery in Oracle utilizing advanced imaging and interchanges as a part of medication (DICOM) form. Paper incorporates the examination of picture recovery methods with the proposed fluffy connectedness picture division joined with geometric minute. Paper additionally gives the usage points of interest of proposed calculation in Oracle. For the examination reason creator executed gimmick extraction systems for shading, surface and shape based peculiarity extraction Connected with Geometric Moments. From the conveyed proposed restorative picture recovery calculation FCISGM gives more exact result when contrasted with Average RGB, Color Moments, Gray Level Co-event Matrix, Local Color Histogram, Global Color Histogram and straightforwardness.

In the paper [3], authors introduced a novel, fast, hybrid and bi-level segmentation technique uniquely developed for segmentation of medical images. Medicinal pictures are by and large described by various locales, and powerless edges. At the point when locales in medicinal pictures are seen as comprised of homogeneous gathering of intensities, it gets to be harder to break down on the grounds that frequently diverse organs or anatomical structures may have comparable dark level or power representation. It has been demonstrated that a precise and quick division of therapeutic pictures is conceivable with our half breed and multilevel division procedure in view of the preparatory results and our work provides for us better results.

The authors in [4] proposed an explicit shape-constrained MAP-MRF-based contour evolution method for the segmentation of organs in 2-D medical images. Creator speaks to the division form expressly as a chain of control focuses. We then give the division issue a role as a shape advancement issue, wherein the development of the form is performed by iteratively comprehending a MAP-MRF marking issue. The advancement of the form is represented by three sorts of earlier data, in particular: (i) appearance former, (ii) limit tenseness earlier, and (iii) shape earlier, each of which is fused as club possibilities into the MAP-MRF issue. We utilize the master-slave double disintegration system to settle the MAP-MRF marking issue in every cycle.

In the paper [5], the authors said that the proposition of their work contained development of portion for the therapeutic pictures by performing the deviation of mapped picture information inside the extent of every area from the piecewise steady model and in light of the regularization term taking into account the capacity of files estimation of the locale. The practical target minimization is completed by two stages minimization in picture division utilizing diagram cut routines, and minimization as for district parameters utilizing consistent point reckoning. Closest neighbor classifiers are acquainted with the benchmarked picture information divided bits. Among the diverse strategies in directed factual example distinguishment, the closest neighbor tenet outcomes in attaining to elite without necessity of the earlier suppositions about the appropriations from which the preparation sets are taken.

In this author [6] proposed new optimization method Pollination Based Optimization (PBO) best optimal clusters in 
color images. The philosophy comprises of four stages: shading space change, era of applicant shading group focuses utilizing Fuzzy K Means, fertilization based improvement technique to choose ideal shading bunch focuses, picture division. Fertilization in blossoms is utilized for selecting ideal groups as a part of shaded pictures. The advancement system functioned admirably on pictures utilized. The aggregate slipped by time used to process division additionally diminished extensively.

\section{IMAGE SEGMENTATION ALGORITHMS}

The human eyes have adjustability for the brightness, which we can only identified dozens of gray-scale at any point of complex image, but can identify thousands of colors. In many cases, only utilize gray-Level information cannot extract the target from background; we must by means of color information. Accordingly, with the rapidly improvement of computer processing capabilities, the color image processing is being more and more concerned by people. The color image segmentation is also widely used in many multimedia applications, for example; in order to effectively scan large numbers of images and video data in digital libraries, they all need to be compiled directory, sorting and storage, the color and texture are two most important features of information retrieval based on its content in the images and video. Therefore, the color and texture segmentation often used for indexing and management of data; another example of multimedia applications is the dissemination of information in the network. Today, a large number of multimedia data streams sent on the Internet, However, due to the bandwidth limitations; we need to compress the data, and therefore it calls for image and video segmentation.

\subsection{Seed Region Growing Algorithm and Watershed Algorithm}

The basic idea of region growing method is a collection of pixels with similar properties to form a region. The steps are as follows:

(i) Find a seed pixel as a starting point for each of needed segmentation.

(ii) Merge the same or similar property of pixel (Based on a pre-determined growing or similar formula to determine) with the seed pixel around the seed pixel domain into the domain of seed pixel.

(iii) These new pixels act as a new seed pixel to continue the above process until no more pixels that satisfy the condition can be included. The seed region growing algorithm is proposed by Adams and Bisch of, Metmert and Jackway [10] further described the dependency relationship between pixels in the seed growth:

(i) The first order of dependence occurs when the number of pixels has the same difference ratio as their vicinity.

(ii) The second order of dependence occurs when a pixels has the same difference ratio as their vicinity Frank and Shouxian Cheng applied the automatic seed selection method, they selected seed which can represents needed segmentation region based on certain similarity criteria and proposed a strategy to solve the above two pixels dependence [1]. The watershed algorithm is more representative in the application of mathematical morphology theory for image segmentation. Watershed algorithm is a region based segmentation techniques image that uses image morphology [2]. Watershed algorithm is an iterative adaptive threshold algorithm. The idea of watershed algorithm is from geography, it see gradient magnitude image as a topographic map, the gradient magnitude in correspond with altitude, the different gradient in correspond with the peak and basin in valley in the image. It sees every object of image (including background) as a separate part and requested there must have one tag at least in the each object (or seed points). Marker is knowledge about the object based on application-oriented; it is selected by the operator manually or by automatic process. The objects can use watershed algorithm to transform and develop regional growth after maker.

\subsection{Gray-scale Image Segmentation}

The segmentation of image raster data into connected regions of common gray-scale has long been seen as a basic operation in image analysis. In texture analysis, just this type of segmentation is possible after individual pixels in an image have been labeled with a numeric classifier. In preparing images for used in geographic information systems (GIs) this segmentation is usually followed by the production of a vector representation for each region. The original algorithm for segmentation, developed by Rosenfeld-Pfalz [3], described a two pass 'sequential algorithm' for the segmentation of binary images. The key feature of the Rosenfeld-Pfalz algorithm is that the image is raster-scanned, first the forward direction, from top left to bottom right, then backwards. During the forward pass, each pixel is located a region label, based on information scanned through; the regions so demarcated may have pixels with more than one label therein. During the backwards pass, a unique label is assigned to each pixel. Hence this classic algorithm can be described as a two pass algorithm. In a previous paper Cohen [4] presented a one-pass algorithm was presented for the segmentation of gray-scale images. Cohen's single pass algorithm proceeds to label pixels on a forward pass, exactly as in Rosenfeld-Phltz, except that during the same forward pass, a lookup-table is prepared, that reveals the connectivity of pixels that have different labels. For most purposes, the labeling of the pixels found the first pass, combined with the look-up-table, provides a complete segmentation, but to actually output an image with unique pixel labels in each region, a pass through the image using the look-up table is required. A new parallel region segmenting and labeling algorithm is available, that is applicable to grayscale images, and is appropriate to coarse scale parallel programming. The key feature of this algorithm is the geometric splitting of the image into rectangular blocks, with one pixel overlap at joins. Then using Cohen's one pass algorithm, each region is separately labeled. Then by examination of the overlap regions, the connectivity of different region labels is determined, through connectivity tables, and finally the overall image is completely segmented into connected domains of common gray-scale. The parallelizable algorithm for the segmentation of gray-scale images involves performing the one-pass algorithm on rectangular sub-images, with a single row or column overlap. What is thus produced is a label for each image pixel, together with connectivity LUT for each region. Then, from the overlap rows and columns produce overlap tables, showing how labels in each region are related.

\subsection{Text Segmentation:}

It is well known that text extraction, including text detection, localization, segmentation and recognition is very important for video auto- understanding. In this paper, we only discuss text segmentation, which is to separate text pixels from complex background in the sub-images from videos. Text segmentation in video images is much more difficult than that in scanning images. Scanning images generally has clean and 
white background, while video images often have very complex background without prior knowledge about the text color. Although there have been several successful systems of video text extraction, few researchers specially study text segmentation in video images deeply. The used strategies could be classified into two main categories: (1) difference (or top-down) and (2) similarity based (or bottom-up) methods. The first methods are based on the foreground background contrast. For example, fixed threshold value method [2], Otsu's adaptive thresholding method [5], global \& local thresholding method [2], Niblack' s method [6]In general, they are simple and fast, but fail when foreground and background are similar. Alternatively, the similarity based methods cluster pixels with similar intensities together. For example, Lienhart uses the split \& merge algorithm [7], Wang et al. combine edge detection, watershed transform and clustering [8]. However, these methods are unstable, since they exploit many intuitive rules about text shape. As an alternative, Chen et al. convert text pixel clustering to labeling problem using Gibbsian EM algorithm [9]. This method is effective but too time consuming. The main problem of most existing methods is that they are sensitive to text color, size, font and background clutter, since they simply exploit either general segmentation method or some prior knowledge. To overcome these problems a new algorithm based on stroke filter is available which discovers the intrinsic characteristics of text and design a robust algorithm especially for text segmentation. In this algorithm stroke filter (SF), describes the intrinsic characteristics of text in terms of scale, orientation and response, a stroke feature based text polarity determination method is available and local region growing method for segmentation refinement based on stroke features and global \& local statistic similarities.

\section{CONCLUSION}

Image segmentation has become a very important task in today's scenario. In the present day world computer vision has become an interdisciplinary field and its applications can be found in any area be it medical, remote sensing, electronics and so on. Thus, to find a appropriate segmentation algorithm based on your application and the type of inputted image is very important. In this paper that we have explained and suggested a few application specific segmentation algorithms which also take into consideration the type of image inputted like color, gray scale and text. In future to achieve better results of segmentation PFCM and $\mathrm{PBO}$ has been optimized using Genetic Algorithm (GA).

\section{REFERENCES}

[1] M. Duraisamy "Cellular Neural Network based on medical image segmentation using Artificial Bee Colony Algorithm”, ISSN 14665986, PP 1 - 6, IEEE, 2014.
[2] Amol Bhagat "Web Based Medical Image Retrieval System Using Fuzzy Connectedness Image Segmentation and Geometric Moments", ISSN 978-1-4799-3010-4/14, IEEE, 2014.

[3] Shihab A. Hameed, "Hybrid and Multilevel Segmentation Technique for Medical Images" 978-14673-6101-9, IEEE, 2013.

[4] Deepak R. Chittajallu "An Explicit Shape Constrained MRF-Based Contour Evolution Method for 2-D Medical Image Segmentation", ISSN 2168-2194, IEEE, 2013.

[5] R. Harini "Image Segmentation Using Nearest Neighbor Classifiers Based On Kernel Formation for Medical Images" ISSN 6745-6754, IEEE, 2013.

[6] Gaganpreet Kaur, "pollination based optimization for color image segmentation" International Journal of Computer Engineering and Technology (IJCET), ISSN 0976 - 6367(Print), ISSN 0976 - 6375 (Online) Volume 3, IEEE, Issue 2, July- September (2012).

[7] Y.YANG, Ch. ZANG "Fuzzy c-means clustering algorithm with a novel penalty term for image segmentation", Regular Issue, OPTO ELECTRONICS REVIEW 13(4),309-315

[8] Othman O. Khalifa, Aisha H. Abdalla, "Multilevel Segmentation Technique for Medical Images" 978-14673-6101-9, IEEE, 2013.

[9] P.K. Nizar Banu "An Analysis of gene expression data using penalized fuzzy c means approach" Department of Computer Applications, B. S. Abdur Rahman University, Chennai, Tamilnadu

[10] N.R. Pal and J.C. Bezdek, "On cluster Validity for the fuzzy c-means model”, IEEE Trans. Fuzzy Systems, Vol. 3, pp. 370-379, 1995.

[11] Gaganpreet Kaur, "pollination based optimization for color image segmentation" International Journal of Computer Engineering and Technology (IJCET), ISSN 0976 - 6367(Print), ISSN 0976 - 6375 (Online) Volume 3, IEEE, Issue 2, July- September (2012).

[12] Vanitha,,"An Effective Biogeography Based Optimization Algorithm to Solve Economic Load Dispatch Problem" Journal of Computer Science 8 ISSN 1482-1486, IEEE, 2012.

[13] Shan Zhu, "A New Diamond Search Algorithm for Fast Matching Motion Estimation, Trans. Image Processing", 9(2000), pp.287-290, IEEE, 2010. 